\title{
Hole states in nanocups in a magnetic field
}

\author{
N. Čukarić, ${ }^{1,2, *}$ V. Arsoski, ${ }^{1, \dagger}$ M. Tadić, ${ }^{1, \ddagger}$ and F. M. Peeters ${ }^{2, \S}$ \\ ${ }^{1}$ School of Electrical Engineering, University of Belgrade, P.O. Box 35-54, 11120 Belgrade, Serbia \\ ${ }^{2}$ Department of Physics, University of Antwerp, Groenenborgerlaan 171, B-2020 Antwerp, Belgium
}

(Received 12 August 2011; revised manuscript received 4 May 2012; published 12 June 2012)

\begin{abstract}
The magnetic-field dependence of the hole states in a nanocup, which is composed of a ring (the nanocup rim) that surrounds a disk (the nanocup bottom), is obtained within the Luttinger-Kohn model for the unstrained $\mathrm{GaAs} /(\mathrm{Al}, \mathrm{Ga}) \mathrm{As}$ and the strained (In,Ga)As/GaAs systems. Aharonov-Bohm oscillations due to angular momentum transitions of the hole ground state appear with periods that vary with the thickness of the disk. The strain in the (In,Ga)As/GaAs nanocup is sensitive to the disk thickness and favors the spatial localization of the heavy holes inside the disk. Therefore, the angular momentum transitions between the valence-band states disappear for much thinner disks than in the case of the unstrained GaAs/(Al, Ga)As nanocups. In both systems, the oscillations in the energy of the hole ground state are found to disappear for thinner inner layer than in the electron ground-state energy. This is due to the different confining potentials and the mixing between the heavyand light-hole states. As a consequence, magnetization of the single hole is found to strongly depend on the bottom thickness of the strained ( $\mathrm{In}, \mathrm{Ga}$ )As/GaAs nanocup. Furthermore, we found that the strain can lead to a spatial separation of the electron and the hole, as in type-II band alignment, which is advantageous for the appearance of the excitonic Aharonov-Bohm effect.
\end{abstract}

DOI: 10.1103/PhysRevB.85.235425

PACS number(s): 73.21.La

\section{INTRODUCTION}

Semiconductor nanorings are a class of systems that are doubly connected. These nanostructures are self-assembled by means of Stranski-Krastanov (SK) growth ${ }^{1-3}$ where first strain leads to lens-shaped quantum dots and subsequently the so-called dewetting process is employed to transform these dots into ringlike structures. The latter leads to the removal of the material from the interior of the dot to the semiconductor matrix. Yet, this process is incomplete and some layer remains inside the (nominal) nanoring opening. ${ }^{4}$ Thus, nanorings formed by SK growth are inherently strained. Alternatively, the droplet epitaxy (DE) technique allows the self-assembly of unstrained GaAs/(Al,Ga)As nanorings. ${ }^{5-7}$ In this method, first the substrate is irradiated by $\mathrm{Ga}$ atoms, which leads to the formation of droplets. These droplets are then crystallized into islands by exposing them to an appropriate flux of $\mathrm{As}_{4}$ molecules. ${ }^{5}$ Similar to the Stranski-Krastanov growth, the DE-fabricated nanorings have complicated shapes and are not fully opened.

Both the SK and DE techniques lead to the formation of nonideal nanorings, the shape of which can be approximated as a cup [see Fig. 1(a)]. The bottom of such a nanocup is a disk that is surrounded by a ring, which is the nanocup's rim. The lateral dimensions of the (In,Ga)As/GaAs nanorings, $R_{1}$ and $R_{2}$, are typically a few nanometers, whereas the width ( $W=R_{2}-R_{1}$ ) of the GaAs/(Al,Ga)As nanorings formed by the droplet epitaxy can be up to a few hundreds of nanometers.

Even though both the SK and DE created rings lack full opening, both these structures are found to exhibit AharonovBohm (AB) oscillations when an external magnetic field threads the ring. ${ }^{2,8}$ The $\mathrm{AB}$ effect is due to the magneticfield-induced change of the phase of the wave function, and in circular rings it manifests itself by transitions between states of different orbital momenta. These transitions and therefore the $\mathrm{AB}$ effect are absent in singly connected quantum dots. ${ }^{9}$ It implies that the electronic structure of a nanocup in a magnetic field changes from ringlike to disklike with increasing thickness of the disk inside the nanocup.

Because of band mixing, the valence-band states in nanorings could be affected by the presence of strain in a more intricate manner than the conduction-band states. For the case of type-II SK-grown quantum dots and quantum-dot molecules, we indeed found that varying the thickness of the dot affects the strain distribution, and in turn the band mixing. ${ }^{11,12}$ Most previous theoretical models of nanorings considered only the electron states in the conduction band. ${ }^{10,13,14}$ Multiband $\mathbf{k} \cdot \mathbf{p}$ calculations of the hole states were previously reported, but they were restricted to the case of fully opened nanorings. ${ }^{15-20}$ The influence of the layer inside the ring opening on the hole states has not been explored to the best of our knowledge. A consequence of the interplay of band mixing and strain might be different magnetic-field dependence of the hole states as compared to the one of the electron states. In addition to the electron and hole states, other important effects that originate from the $\mathrm{AB}$ effect, such as persistent currents and orbital magnetism, ${ }^{8,10}$ could also be affected by the presence of the disk inside the nanocup.

Let us briefly note that in addition to the electron and hole energy levels, the exciton energy levels should also be affected when increasing the disk thickness in the nanocup. The exciton ground-state energy exhibits oscillations with the magnetic field, which is called the excitonic Aharonov-Bohm effect. ${ }^{21-23}$ This effect has been sought in three-dimensional (3D) nanorings for some time, ${ }^{24}$ whereas it only recently has been observed in (In,Ga)As/GaAs nanorings. ${ }^{25,26}$ The theory revealed that the presence of strain is an important ingredient for the appearance of excitonic $\mathrm{AB}$ oscillations in type-I nanorings. ${ }^{27}$

In this paper, we compute and analyze the electron and hole states in a nanocup, the geometry and characteristic dimensions of which are displayed in Fig. 1(a), where the disk is shown by blue color, and the ring by red color. $R_{1}$ denotes the radius of the disk, $t$ is the thickness of the disk, $R_{2}$ 
(a)

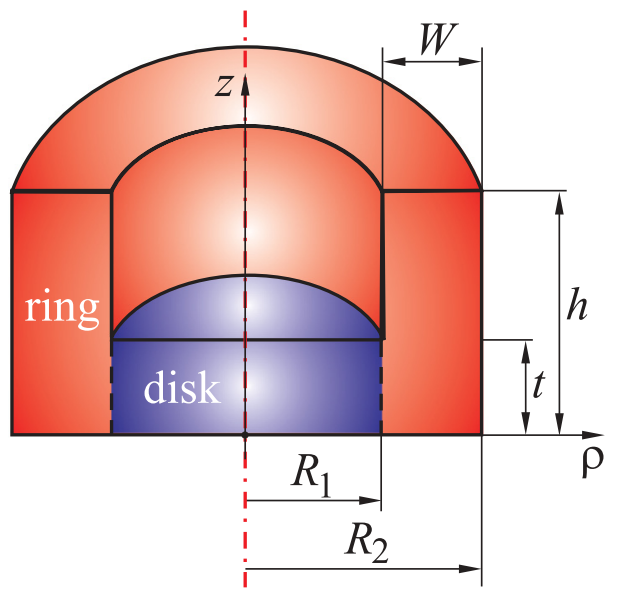

(b)

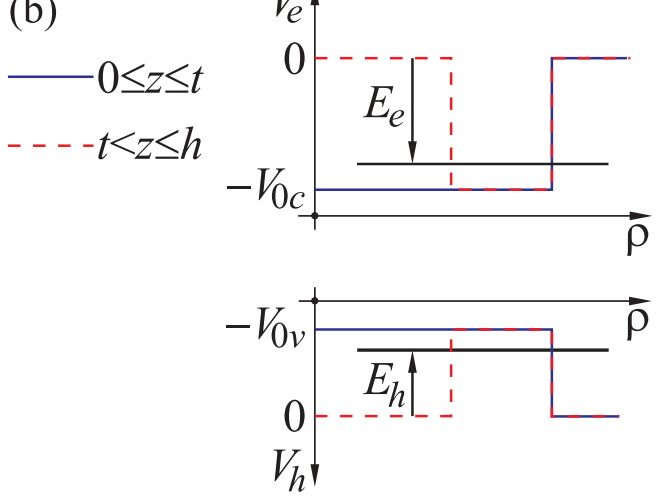

FIG. 1. (Color online) (a) The cross section through the center of the nanocup, which is composed of a disk (blue color) with thickness $t$ and a ring (red color), the height of which is denoted by $h$. (b) Variation of the conduction- and valence-band edges in the nanocup along the $\rho$ direction at height $0 \leqslant z \leqslant t$ (solid blue line) and $t<z \leqslant h$ (dashed red line).

is the outer radius of the ring, $W=R_{2}-R_{1}$ is the width of the ring, and $h$ is its height. For $t=0$, the nanocup becomes a nanoring. Previously, different analytical functions were adopted to model the shape of the fabricated rings. For the SK-grown rings, Fomin et al. used a function which takes into account both the extension of the ring material in the opening and the deviation from axial symmetry in the $x y$ plane. ${ }^{13}$ More recently, Thu et al. described the shape of the DE-formed nanorings by overlapping triple concentric nanorings,${ }^{14}$ which is more complex than our nanocup. But, the existence of the $\mathrm{AB}$ effect is related to the topology rather than the exact shape of the structure. Furthermore, effects of the inner layer on the nanocup electronic structure are expressed through a single parameter: the disk thickness $t$.

Here, we investigate how a perpendicular magnetic field affects the hole states in the nanocup. Furthermore, the effects of strain in the electronic structure are explored by (1) comparing the electron and the hole energy levels in the (In,Ga)As/GaAs nanocup and (2) comparing the hole states in the strained SK-fabricated (In,Ga)As/GaAs nanocup with those in the unstrained DE-formed GaAs/(Al,Ga)As. The strain distribution in the (In,Ga)As/GaAs nanocups is determined from the model of isotropic strain using the inclusion theory. ${ }^{28,29}$ The effects due to varying the disk thickness on the angular momentum transitions and mixing between the hole states in the nanocup are particularly explored. Even though the DE-formed GaAs/(Al,Ga)As nanocups are in general larger, for comparative purposes, we assume here that their dimensions are equal to those of the (In,Ga)As/GaAs cups. The four-band Luttinger-Kohn model is employed to compute the valence-band states, whereas the electronic structure of the conduction band is determined from the single-band effective mass theory. A similar model was recently employed to model the zero-magnetic-field electron and hole states in unstrained GaAs/(Al,Ga)As nanocups of larger size, which is typical for experimentally realized nanocups made by means of the droplet epitaxy. ${ }^{30}$ We also analyze how the presence of the disk in the nanocup affects the magnetization due to the single-particle states. ${ }^{31}$ The influence of the geometry of the structure on the electron and hole energy levels is briefly discussed. Finally, we note that our calculations do not aim to analyze any particular sample of currently fabricated nanorings. Nevertheless, the present model could provide a qualitative description of the electronic structure of experimental nanorings where the layer is present inside the ring opening.

The paper is organized as follows. In Sec. II, we present the model used to obtain the electronic structure. The results of our numerical calculations are presented and discussed in Sec. III. Our conclusions are given in Sec. IV.

\section{THEORETICAL MODELS}

\section{A. Model of elasticity}

The analyzed (In, Ga)As/GaAs cup is strained due to the lattice mismatch between (In,Ga)As and GaAs, which results in changes in the effective potentials for the electrons and the holes. The strain distribution is determined by the approach of Downes, which applied the Eshelby inclusion theory under the approximation of isotropic elasticity. ${ }^{28,29}$ The component of the strain tensor $\varepsilon_{i j}$ in this theory is given by

$$
\varepsilon_{i j}(\mathbf{r})=-\varepsilon_{0} \Theta(\mathbf{r})-\frac{\varepsilon_{0}}{4 \pi} \frac{1+v}{1-v} \oint_{S^{\prime}} \frac{\left(x_{i}-x_{i}^{\prime}\right)}{\left|\mathbf{r}-\mathbf{r}^{\prime}\right|^{3}} d S_{j}^{\prime} .
$$

Here, $\varepsilon_{0}$ denotes the lattice mismatch between the cup and the matrix $\varepsilon_{0}=\left(a_{d}-a_{m}\right) / a_{m}, \Theta$ is unity inside the cup and zero otherwise, the integration is over the surface of the cup $S^{\prime}$, and $v$ is the Poisson ratio which is taken to be $1 / 3$. It is straightforward to show that the hydrostatic strain is

$$
\varepsilon_{\text {hyd }}=\sum_{i} \varepsilon_{i i}= \begin{cases}-\varepsilon_{0} & \text { in the cup, } \\ 0 & \text { in the matrix. }\end{cases}
$$

The effective potentials for the heavy holes, light holes, and electrons $V_{h h}, V_{l h}$, and $V_{e}$, respectively, are given by ${ }^{11,12}$

$$
\begin{aligned}
V_{h h}(\mathbf{r})= & a_{v}\left(\varepsilon_{x x}+\varepsilon_{y y}+\varepsilon_{z z}\right)+b\left(\varepsilon_{x x} / 2+\varepsilon_{y y} / 2-\varepsilon_{z z}\right) \\
& +V_{\text {off }, h}(\mathbf{r}), \\
V_{l h}(\mathbf{r})= & a_{v}\left(\varepsilon_{x x}+\varepsilon_{y y}+\varepsilon_{z z}\right)-b\left(\varepsilon_{x x} / 2+\varepsilon_{y y} / 2-\varepsilon_{z z}\right) \\
& +V_{\text {off }, h}(\mathbf{r}), \\
V_{e}(\mathbf{r})= & a_{c}\left(\varepsilon_{x x}+\varepsilon_{y y}+\varepsilon_{z z}\right)+V_{\mathrm{off}, e}(\mathbf{r}),
\end{aligned}
$$


where $a_{c}, a_{v}$, and $b$ denote the deformation potentials, and $V_{\text {off }, e}$ and $V_{\text {off }, h}$ are the confining potentials due to the offsets between the valence and the conduction bands, respectively. All three effective potentials are axially symmetric for the analyzed axially symmetric nanocup. The shape of $V_{\mathrm{off}, e}$ and $V_{\text {off, } h}$, and the adopted orientations of the energy axes are sketched in Fig. 1(b). Because strain is absent in the $\mathrm{GaAs} /(\mathrm{Al}, \mathrm{Ga})$ As nanocup $\left(\varepsilon_{x x}=\varepsilon_{y y}=\varepsilon_{z z}=0\right)$, we have in this case $V_{h h}=V_{l h}=V_{\text {off }, h}$ and $V_{e}=V_{\text {off }, e}$. The energies of the band extrema in the matrix far away from the nanocup boundary, where full strain relaxation is achieved, are taken as the reference energies for the effective potentials. Furthermore, $V_{\text {off }, h}=-V_{0 v}$ and $V_{\text {off }, e}=-V_{0 c}$ in the cup, where $V_{0 v}$ and $V_{0 c}$ denote the valence- and conduction-band offsets, respectively.

\section{B. Hole and electron states}

For the hole states in the analyzed nanocup, the LuttingerKohn (LK) Hamiltonian in the axial approximation and for the case of a uniform magnetic field oriented along the $z$ direction reads as ${ }^{11,32}$

$$
H_{h}=\frac{\hbar^{2}}{2 m_{0}}\left[\begin{array}{cccc}
H_{h h} & R & S & 0 \\
R^{+} & H_{l h} & 0 & S \\
S^{+} & 0 & H_{l h} & -R \\
0 & S^{+} & -R^{+} & H_{h h}
\end{array}\right]+\kappa \mathcal{J}_{z} H_{z}+V_{h} .
$$

The matrix elements of the kinetic part of the LK Hamiltonian are given by

$$
\begin{aligned}
H_{h h} & =\left(\gamma_{1}+\gamma_{2}\right)\left(k_{x}^{2}+k_{y}^{2}\right)+\left(\gamma_{1}-2 \gamma_{2}\right) k_{z}^{2}, \\
H_{l h} & =\left(\gamma_{1}-\gamma_{2}\right)\left(k_{x}^{2}+k_{y}^{2}\right)+\left(\gamma_{1}+2 \gamma_{2}\right) k_{z}^{2}, \\
R & =-2 \sqrt{3} \gamma_{3} k_{z} k_{-}, \\
S & =-\sqrt{3} \frac{\gamma_{2}+\gamma_{3}}{2} k_{-}^{2}, \\
H_{z} & =i \frac{\hbar^{2}}{m_{0}}\left[k_{x}, k_{y}\right]=\hbar \omega_{c} .
\end{aligned}
$$

Here, $\gamma_{1}, \gamma_{2}, \gamma_{3}$, and $\kappa$ denote the Luttinger parameters, $\omega_{c}$ is the cyclotron frequency, and $V_{h}$ the potential part of the LK Hamiltonian, which in our model is given by the diagonal matrix

$$
V_{h}=\operatorname{diag}\left(V_{h h}, V_{l h}, V_{l h}, V_{h h}\right) .
$$

$\mathcal{J}_{z}$ is the matrix of the $z$ projection of the Bloch angular momentum $J_{z}$,

$$
\begin{aligned}
\mathcal{J}_{z} & =\operatorname{diag}\left(j_{z 1}, j_{z 2}, j_{z 3}, j_{z 4}\right) \\
& =\operatorname{diag}(+3 / 2,+1 / 2,-1 / 2,-3 / 2),
\end{aligned}
$$

and $k_{x}, k_{y}$, and $k_{z}$ are the hole wave-vector projections on the Cartesian axes,

$$
\begin{aligned}
& k_{x}=-i \frac{\partial}{\partial x}-\frac{e B y}{2 \hbar} \\
& k_{y}=-i \frac{\partial}{\partial y}+\frac{e B x}{2 \hbar}, \\
& k_{z}=-i \frac{\partial}{\partial z}
\end{aligned}
$$

Because of axial symmetry, the $z$ projection of the total angular momentum $F_{z}=L_{z}+J_{z}$ commutes with the Hamiltonian, and the quantum number of the total angular momentum $f_{z}$ $\left(F_{z}=f_{z} \hbar\right)$ is a good quantum number. Equation (4) is written for the case of the position-independent Luttinger parameters, which are assumed to correspond to the material of the nanocup, where the hole is mainly localized. Note that strain is taken into account in only the diagonal matrix elements of the Hamiltonian. The off-diagonal matrix elements depend on the shear strain tensor components and the difference $\varepsilon_{x x}-\varepsilon_{y y}{ }^{33}$ For the case of nanodisks, we found that the shear strain-tensor components are localized in the small regions close to the dot boundary. ${ }^{11}$ Similarly, the difference between $\varepsilon_{x x}$ and $\varepsilon_{y y}$ is small in the axial geometry. Hence, the off-diagonal matrix elements could be neglected, which along with the axial approximation of the kinetic part of the Hamiltonian makes the model axially symmetric. ${ }^{11}$

A similar Hamiltonian has been recently employed to analyze the coupling between the hole states in a system of two vertically coupled nanodisks. ${ }^{34}$ The results from the LK Hamiltonian were compared with those of a model which contains magnetic terms in only the main diagonal. ${ }^{19,20}$ The latter model was constructed by employing substitution $\mathbf{p} \rightarrow \mathbf{p}-e \mathbf{A}$ in the Schrödinger equation prior the envelope approximation, which was not the case in the Luttinger-Kohn model. Therefore, the off-diagonal terms due to magnetic field are removed in the Hamiltonian of Ref. 19. Quite interestingly, even though the latter model is an approximate approach, it leads to splitting of the energy levels in the magnetic field, which is qualitatively similar to the experimental data. ${ }^{34}$ Therefore, such a comparison indicated that the off-diagonal magnetic terms in the original Luttinger-Kohn model are too large when applied to a nanodisk. Similar to the nanodots previously analyzed by the approximate Hamiltonian, the removal of the off-diagonal elements due to magnetic field in the multiband model of the nanocup is expected to reduce the general (increasing or decreasing) trend of the hole ground-state variation with the magnetic field. However, the magnetic off-diagonal terms could not have a large influence on the positions of the angular momentum transitions between the hole states of different total orbital momenta, as Ref. 20 demonstrated for the case of the nanoring. Moreover, the model of Ref. 19 relies on an ad hoc approach, whereas the LK model of the hole states in a magnetic field is a consistent procedure, which successfully explained the valence-band states of various semiconductors and their nanostructures in a magnetic field. Its restrictions are basically related to the size of the system and the influence of the interface. Furthermore, the hole states in a nanostructure should tend towards the bulk states when the size of the system increases. In the approximate theory of Ref. 19, this could be difficult to achieve because mixing due to magnetic field with states outside the basis set is not taken into account.

The LK Hamiltonian acts on the Luttinger spinor $\Psi_{h}$, which contains the envelope functions of the heavy and light holes $\Psi_{h}=\left[\Psi_{1}, \Psi_{2}, \Psi_{3}, \Psi_{4}\right]^{T}$. Here, the envelope functions $\Psi_{1}$ and $\Psi_{4}$ correspond to the heavy holes, while $\Psi_{2}$ and $\Psi_{3}$ are the light-hole envelope functions. Each envelope function in the 
Luttinger spinor has the form

$$
\Psi_{k}(\varphi, \rho, z)=\frac{1}{\sqrt{2 \pi}} \psi_{k}(\rho, z) e^{i l_{k} \varphi},
$$

where $l_{k}=f_{z}-j_{z k}$ is the orbital quantum number corresponding to the envelope function $\psi_{k}$. For a given $f_{z}$, the set of orbital quantum numbers $\left(l_{1}, l_{2}, l_{3}, l_{4}\right)$ corresponds to the Luttinger spinor $\Psi_{h}$. For example, the set of orbital quantum numbers $(0,1,2,3)$ correspond to the envelope functions in the $f_{z}=+3 / 2$ hole state. The hole states are labeled by $n|L|_{f_{z}}$, where $n$ denotes the principal quantum number, and $L$ denotes the minimum value of $l_{k}$ in the Luttinger spinor, ${ }^{11,32}$ i.e., $L=\min \left(\left|l_{k}\right|\right)$.

In order to solve the eigenvalue problem $H \Psi=E \Psi$, each $\psi_{k}$ is expanded into products of the $\rho$-dependent Bessel functions of the first kind $J_{l}$ and the $z$-dependent $\sin / \cos$ function:

$$
\begin{aligned}
\psi_{k}(\rho, z)= & \sqrt{\frac{2}{H}} \sum_{n_{\rho}=1}^{N_{\rho}} A_{n_{\rho} l_{k}} J_{l_{k}}\left(\mu_{n_{\rho} l_{k}} \frac{\rho}{R}\right) \\
& \times\left[\sum_{n_{z}=1}^{N_{z}} a_{n_{z} k} \cos \left(\left(2 n_{z}-1\right) \pi \frac{z}{H}\right)\right. \\
& \left.+\sum_{n_{z}=1}^{N_{z}} b_{n_{z} k} \sin \left(2 n_{z} \pi \frac{z}{H}\right)\right] .
\end{aligned}
$$

Here, $R$ and $H$ denote the radius and the height of the simulation box, respectively, $A_{n_{\rho} l_{k}}$ is the normalization factor of the Bessel functions, $a_{n_{z} k}$ and $b_{n_{z} k}$ are the expansion coefficients, and $\mu_{n_{\rho} l_{k}}$ is the $n_{\rho}$ th zero of the Bessel function of $l_{k}$ th order.

The electronic structure of the conduction band is extracted from the single-band effective-mass equation

$$
\begin{aligned}
H_{e} \Psi_{e}= & \left(-\frac{\hbar^{2}}{2 m} \nabla^{2}-\frac{i \hbar e B}{2 m} \frac{\partial}{\partial \varphi}+\frac{(e B \rho)^{2}}{8 m}\right. \\
& \left. \pm \frac{g_{\text {eff }}}{2} \mu_{B} B+V_{e}(\mathbf{r})\right) \Psi_{e}=E \Psi_{e}
\end{aligned}
$$

Here, $m$ denotes the effective mass of the electron in the conduction band, $\mu_{B}$ is the Bohr magneton, and $V_{e}(\mathbf{r})=$ $V_{e}(\rho, z)$. The plus and minus sign in the fourth term in parentheses correspond to spin up and spin down, respectively. The Hamiltonian is axially symmetric, thus, the $z$ projection of the orbital momentum $L_{z}$ commutes with the Hamiltonian and the electron states are classified according to the orbital quantum number $l$. The electron envelope function $\Psi_{e}$ has the same form as the hole envelope functions $\Psi_{k}$ [see Eq. (9)]. Furthermore, the single-band effective-mass equation is solved by expanding $\psi_{e}$ in the basis employed to compute the hole states. For convenience, the energy of the electron ground state is denoted by $E_{(1)}^{X}$, where $X$ labels the nanocup: $X=\mathrm{GaAs}$ for the GaAs/(Al,Ga)As nanocup and $X=\mathrm{InGaAs}$ for the (In,Ga)As/GaAs nanocup.

\section{NUMERICAL RESULTS}

We consider nanocups composed of (1) GaAs surrounded by $\mathrm{Al}_{0.3} \mathrm{Ga}_{0.7}$ As and (2) $\mathrm{In}_{0.5} \mathrm{Ga}_{0.5}$ As in a GaAs matrix. The compositions of $(\mathrm{Al}, \mathrm{Ga}) \mathrm{As}$ and $(\mathrm{In}, \mathrm{Ga}) \mathrm{As}$ are taken close to the experimental values. ${ }^{7,10}$ Furthermore, both cups are chosen to have equal dimensions: height $h=5 \mathrm{~nm}$, the inner radius $R_{1}=8 \mathrm{~nm}$, and the outer radius $R_{2}=15 \mathrm{~nm}$. These dimensions correspond to the experimental (In,Ga)As cups, whereas the GaAs cups are typically larger and their shape differs from the (In,Ga)As cups. ${ }^{7,10,25}$ Nevertheless, our aim is to estimate how strain affects the electron and hole states in the nanocup, and to resolve these effects from the effects of varying size when the disk thickness varies. The Luttinger parameters, the band offsets, the deformation potentials, and the effective masses are all taken from Ref. 35. The effective Landé $g$ factor is taken to be equal $g_{\text {eff }}=-0.44$, and the $\kappa$ Luttinger parameter equals $\kappa=1.72 .{ }^{36}$ These values correspond to GaAs, but in the (In,Ga)As nanodots, the measured Zeeman splitting is much smaller than what is expected from the bulk values of $g_{\text {eff }}$ and $\kappa$. The values estimated from such measurements are in fact closer to the experimental values for the GaAs matrix, ${ }^{37}$ therefore, we adopt the same $g_{\text {eff }}$ and $\kappa$ in both analyzed nanocups.

In order to compare the electronic structure of the analyzed 3D ring with the one of the one-dimensional (1D) ring, we compute a few characteristics of the electron and hole states in the analyzed nanocups. First, we note that the orbital momentum transitions in the $1 \mathrm{D}$ rings correspond to the magnetic flux $\Phi=(i-1 / 2) \Phi_{0}\left(\Phi_{0}=h / e\right.$ is the flux quantum; $i=1,2,3, \ldots)$ through the ring. ${ }^{8}$ Therefore, we define the effective radius of the equivalent $1 \mathrm{D}$ ring,

$$
R_{\mathrm{eff}, i}=\sqrt{(i-1 / 2) \Phi_{0} /\left(\pi B_{i}\right)}, \quad i=1,2,3, \ldots
$$

where $B_{i}$ is the magnetic field of the $i$ th angular momentum transition in the analyzed 3D nanocup. Second, the probability density of the hole in the ground state is computed as

$$
D_{h}=\sum_{k=1}^{4}\left|\Psi_{k}\right|^{2}
$$

Third, the magnetization due to the single electron and the single hole is computed as

$$
M=\mp \frac{\partial E_{1}}{\partial B} .
$$

Here, $E_{1}$ is the ground-state energy, the upper sign is for the electron, and the lower sign for the hole.

\section{A. Effective potentials}

Contour plots of the effective potentials in the conduction, heavy-hole, and light-hole bands in the (In,Ga)As/GaAs nanocup are displayed in Fig. 2. The upper panel in Fig. 2 [Figs. 2(a)-2(c)] displays the effective potentials in the nanoring $(t=0)$, the middle panel [Figs. 2(d)-2(f)] shows the effective potentials in the nanocup with a $t=2 \mathrm{~nm}$ thick disk, and the lower panel [Figs. 2(g)-2(i)] shows the effective potentials for $t=4 \mathrm{~nm}$. The effective potentials in the conduction band $V_{e l}$ depend on the hydrostatic strain, and are therefore steplike in all three cases displayed in Fig. 2 [see Figs. 2(a), 2(d), and 2(g)]. Increase of the disk thickness does not affect the hydrostatic strain, thus, the conductionband states in the (In,Ga)As/GaAs and the GaAs/(Al,Ga)As 

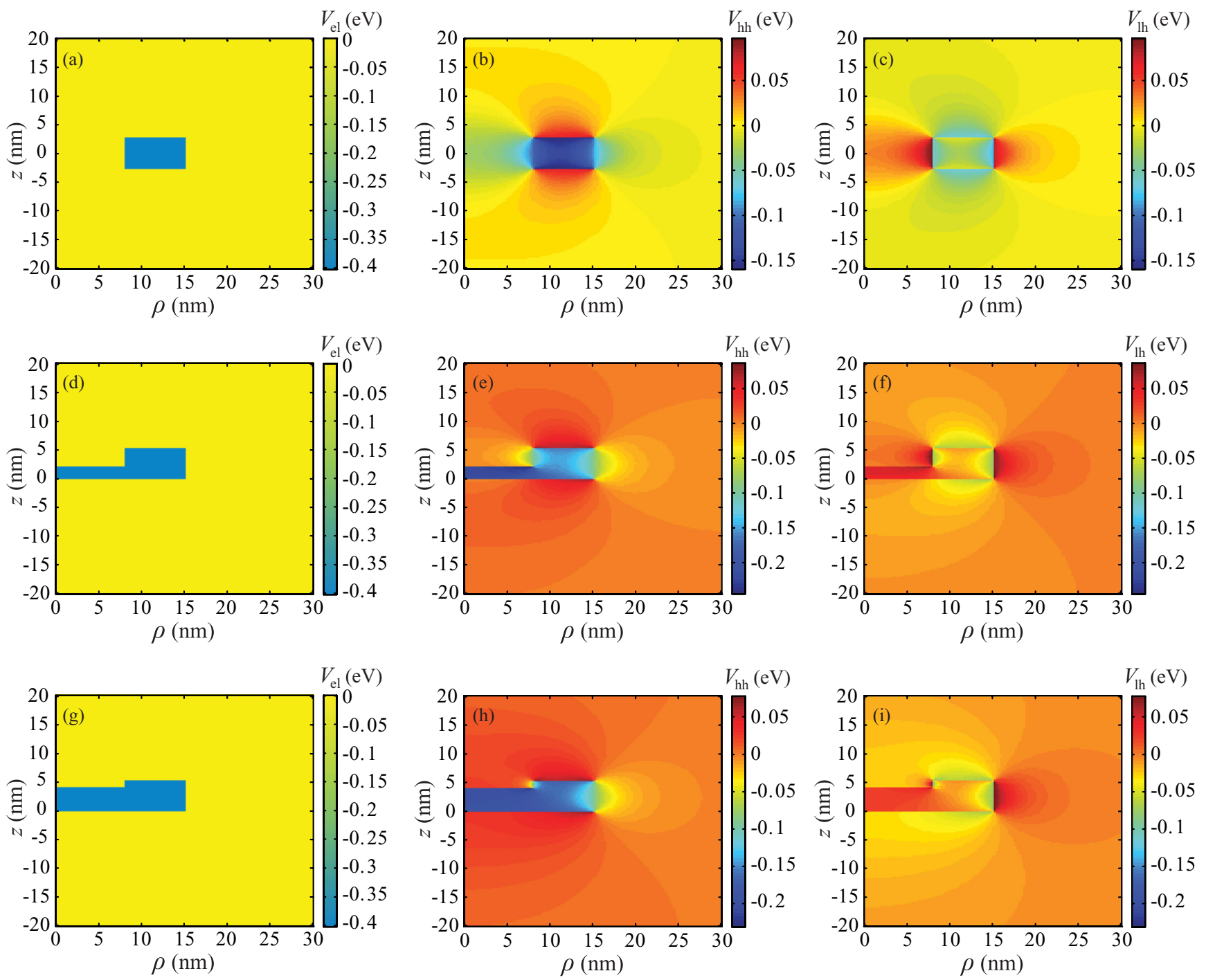

FIG. 2. (Color online) The effective potentials for the single-particle states in the (In,Ga)As/GaAs nanocup as they vary with the disk thickness. Upper panel: The case of a nanoring $(t=0)$ : (a) $V_{e l}$, (b) $V_{h h}$, and (c) $V_{l h}$. Middle panel: The nanocup with a $t=2 \mathrm{~nm}$ thick disk: (d) $V_{e l}$, (e) $V_{h h}$, and (f) $V_{l h}$. Lower panel: The nanocup with a $t=4 \mathrm{~nm}$ thick disk: (g) $V_{e l}$, (h) $V_{h h}$, and (i) $V_{l h}$.

nanocups are similarly affected by the presence of the disk inside the ring opening.

Because of both the hydrostatic and tetrahedral deformations of the structure, the effective potentials for the heavy and light holes are different. For the case of the nanoring $(t=0)$, the effective potential well for the heavy hole inside the ring is deeper than the effective potential well for the light hole, as Figs. 2(b) and 2(c) show. Furthermore, the shallow confining potential wells for the heavy hole extend laterally, whereas a similar confining potential for the light hole extends vertically above and below the nanoring. However, because of the shallower effective potential well inside the ring, the light hole is less confined than the heavy hole.

For the case of a finite $t$, a superposition of strain fields inside the disk and around the ring produces a deeper effective potential well for the heavy hole inside the disk than in the ring [see Figs. 2(e) and 2(h)]. Therefore, strain favors confinement of the heavy hole in the disk. When $t$ increases from 2 to 4 $\mathrm{nm}$, no large changes in $V_{h h}$ are observed [compare Figs. 2(e) and 2(h)]. On the other hand, the effective potential barrier for the light hole in the nanocup increases, except close to the outer boundary of the nanocup, as Figs. 2(f) and 2(i) show. When $t / h$ approaches unity, the regions of confining $V_{l h}$ above and below the ring extend towards the cup center, which establishes the confinement of the light hole in the matrix around the cup boundary, as for a disk. ${ }^{11,12}$

\section{B. Conduction-band states}

The energy levels in the conduction band of the GaAs/(Al,Ga)As nanocup with a $t=0,2 \mathrm{~nm}$, and $4 \mathrm{~nm}$ thick disk are shown in Figs. 3(a)-3(c), respectively. The orbital momentum of the electron ground state is explicitly shown, and the points where the different $l$ states cross in the electron ground state are connected by thin dashed lines in the different diagrams. For comparison, variations of the energy of the electron ground state in the (In,Ga)As/GaAs nanocup $E_{(1)}^{\mathrm{InGaAs}}$ with the magnetic field are shown by the thick dashed lines. 


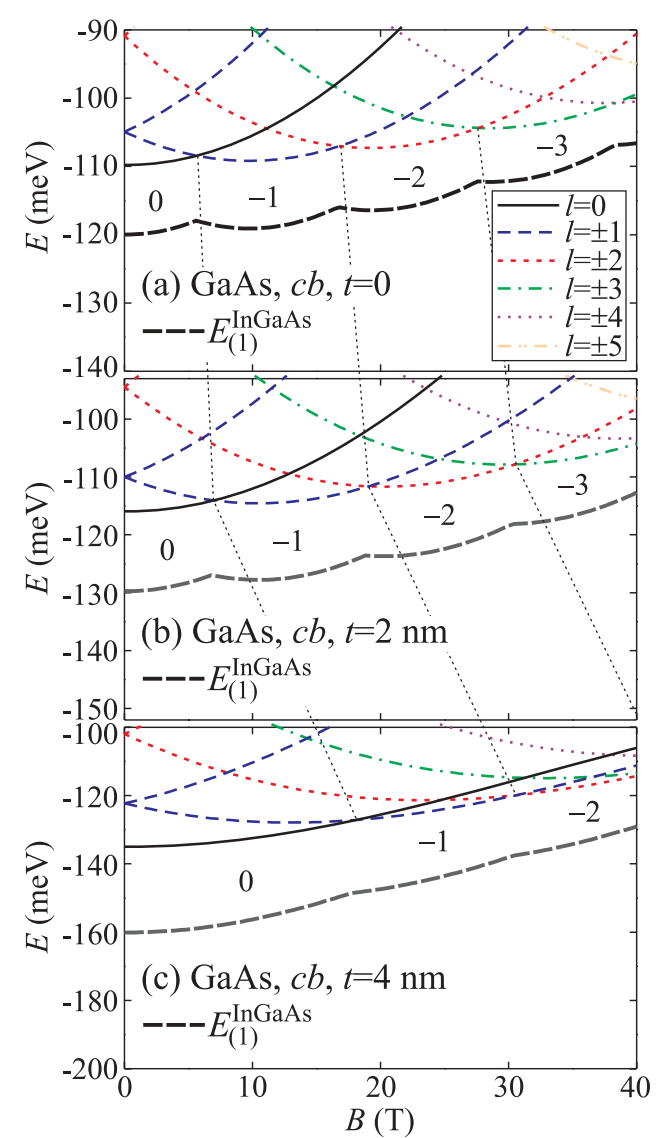

FIG. 3. (Color online) The lowest-energy spin-up electron energy levels for orbital momenta $\left|l_{e}\right| \leqslant 5$ in the GaAs nanocup as a function of the magnetic field for (a) $t=0$, (b) $t=2 \mathrm{~nm}$, and (c) $t=4 \mathrm{~nm}$. The orbital momentum of the ground $c b$ state is explicitly denoted in each diagram, and the crossings of the different $l$ states are joined by the thin dashed lines. The thick dashed line is the energy of the electron ground state in the (In,Ga)As/GaAs nanocup.

For convenience, the values of $E_{(1)}^{\mathrm{InGaAs}}$ in Fig. 3 are increased by $75 \mathrm{meV}$. For all three values of the disk thickness, both $E_{(1)}^{\mathrm{GaAs}}$ and $E_{(1)}^{\mathrm{InGaAs}}$ vary oscillatory with the magnetic field due to the orbital momentum transitions. The crossings between different $l$ states in the nanoring [see Fig. 3(a)] are almost periodic with the magnetic field, as in 1D rings, where the orbital momentum transitions are odd multiplicators of half of the flux quantum divided by the area of the ring. ${ }^{8}$ From the value of the magnetic field of the first and the second orbital momentum transition in the analyzed nanoring, $B_{1}=5.6 \mathrm{~T}$ and $B_{2}=16.8 \mathrm{~T}$, the radii of the equivalent $1 \mathrm{D}$ rings amount to $R_{\mathrm{eff}, 1}=R_{\mathrm{eff}, 2}=\sqrt{\Phi_{0} / 2 \pi B_{1}}=10.8 \mathrm{~nm}$, which is close to the average ring radius $\left(R_{1}+R_{2}\right) / 2=11.5 \mathrm{~nm}$.

When the disk thickness increases, the orbital momentum transitions shift towards larger values, as the thin dashed lines joining the orbital momentum transitions in the three diagrams indicate in Fig. 3. These shifts are almost constant, which is demonstrated by nearly parallel thin dashed lines. For $t=2 \mathrm{~nm}$, which is $40 \%$ of the ring height, the orbital momentum transitions shift negligibly from the $t=0$ case [compare Figs. 3(a) and 3(b)]. But, when $t$ increases to $4 \mathrm{~nm}$ (80\% of the ring height), the orbital momentum transitions shift by about 13 T [compare Figs. 3(a) and 3(c)]. Moreover,

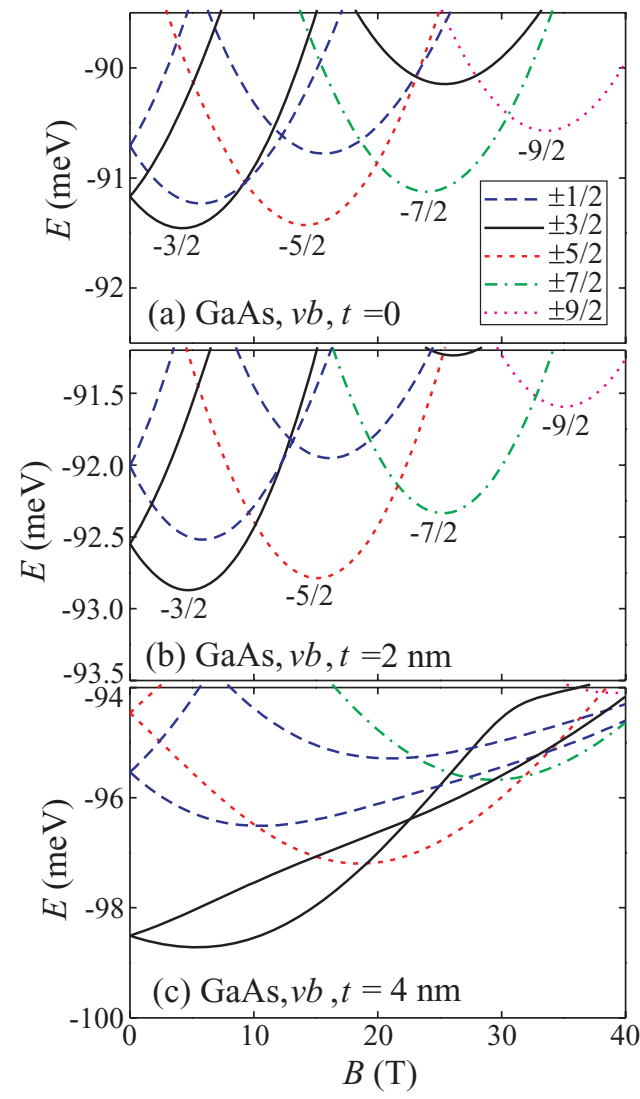

FIG. 4. (Color online) The energy levels in the valence band of the GaAs/(Al,Ga)As nanocup for (a) the $t=0$, (b) $t=2 \mathrm{~nm}$, and (c) $t=4 \mathrm{~nm}$ thick disk. The $z$ projection of the total angular momentum is explicitly denoted in the figures.

increasing the confinement inside the disk favors the low $|l|$ states, therefore, $R_{\mathrm{eff}, 1}$, which is determined from the crossing between the $l=0$ and -1 electron energy levels, becomes much smaller than $R_{\mathrm{eff}, k}$ for $k>1$. The ground-state energy level in the (In,Ga)As/GaAs nanocup varies with $B$ similarly to the $\mathrm{GaAs} /(\mathrm{Al}, \mathrm{Ga}) \mathrm{As}$ nanocup, as the thick dashed lines depict in Fig. 3. Hence, a modification of the material parameters does not produce any qualitative change of the obtained energy spectra in the conduction band.

\section{Valence-band states}

The zero-magnetic-field hole ground state in the $\mathrm{GaAs} /(\mathrm{Al}, \mathrm{Ga}) \mathrm{As}$ nanocup is found to be in the total angular momentum state $f_{z}=-3 / 2$ irrespective of the value of $t$, as is clear from Figs. 4(a)-4(c) for $t=0,2 \mathrm{~nm}$, and $4 \mathrm{~nm}$, respectively. The $f_{z}=-3 / 2$ state is dominated by the heavyhole envelope function with $l=0$. When $B$ increases, the states of different $f_{z}$ cross in the ground states, but because of the band mixing $B_{1}$ is shifted with respect to $B_{1}$ for the electron ground state [compare Figs. 3(a) and 4(a)]. However, the other angular momentum transitions that occur in the ground state are separated by almost fixed intervals of $B$. Such a similarity to the conduction-band states could be ascribed to arise from similar effective potentials for the electrons and heavy and light holes. The angular momentum transitions are associated with the change of the orbital momenta of all the components 


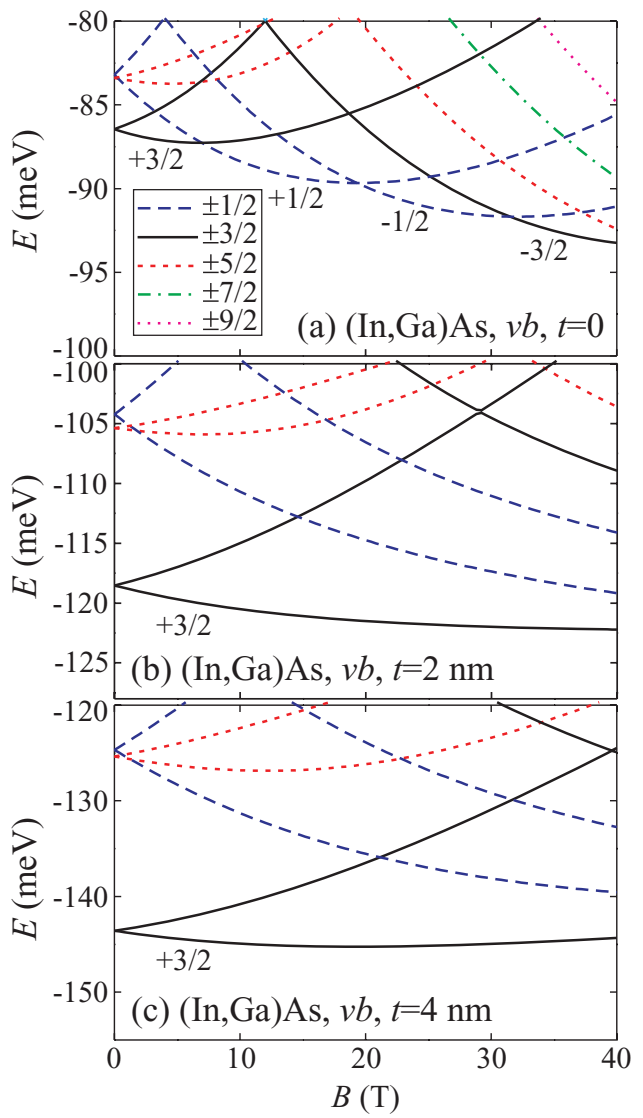

FIG. 5. (Color online) The same as Fig. 4, but now for the (In,Ga)As/GaAs nanocups.

of the Luttinger spinor by -1 . Furthermore, similar to the conduction band, the angular momentum transitions between the valence-band states do not shift much when $t$ increases from 0 to $2 \mathrm{~nm}$, as shown in Figs. 4(a) and 4(b). But, when $t$ increases to $4 \mathrm{~nm}$, those shifts become considerable [see Fig. 4(c)].

The variations of the hole states with magnetic field in the (In,Ga)As/GaAs nanoring (the $t=0$ case) shown in Fig. 5(a) are similar to those presented in Ref. 20. Also, they are similar to the orbital momentum transitions in the conduction band (see Fig. 3). However, they are arranged differently than the angular momentum transitions in the $\mathrm{GaAs} /(\mathrm{Al}, \mathrm{Ga}) \mathrm{As}$ nanoring, which was shown in Fig. 4. It indicates that mixing is reduced in the (In,Ga)As/GaAs nanorings, which indeed occurs due to different effective potentials of the heavy and light holes in a strained system. Furthermore, because of the large difference between the Luttinger parameters, the hole ground-state energy levels in the two systems exhibit opposite trends with $B$ [compare Figs. 4(a) and 5(a)]. For nonzero value of $t$, strain favors confinement of the heavy hole in the disk, as is apparent from Figs. 5(b) and 5(c) for $t=2 \mathrm{~nm}$ and $4 \mathrm{~nm}$, respectively. The deeper effective potential well in the disk as compared to the ring causes the heavy-hole states to be mainly localized in the disk, which favors the low $|l|$ states. As a consequence, the $1 S_{+3 / 2}$ state, which has the $l=0$ heavy-hole component, is the hole ground state in the whole investigated range of $B$, i.e., from 0 to $40 \mathrm{~T}$. On the other hand, because the effective
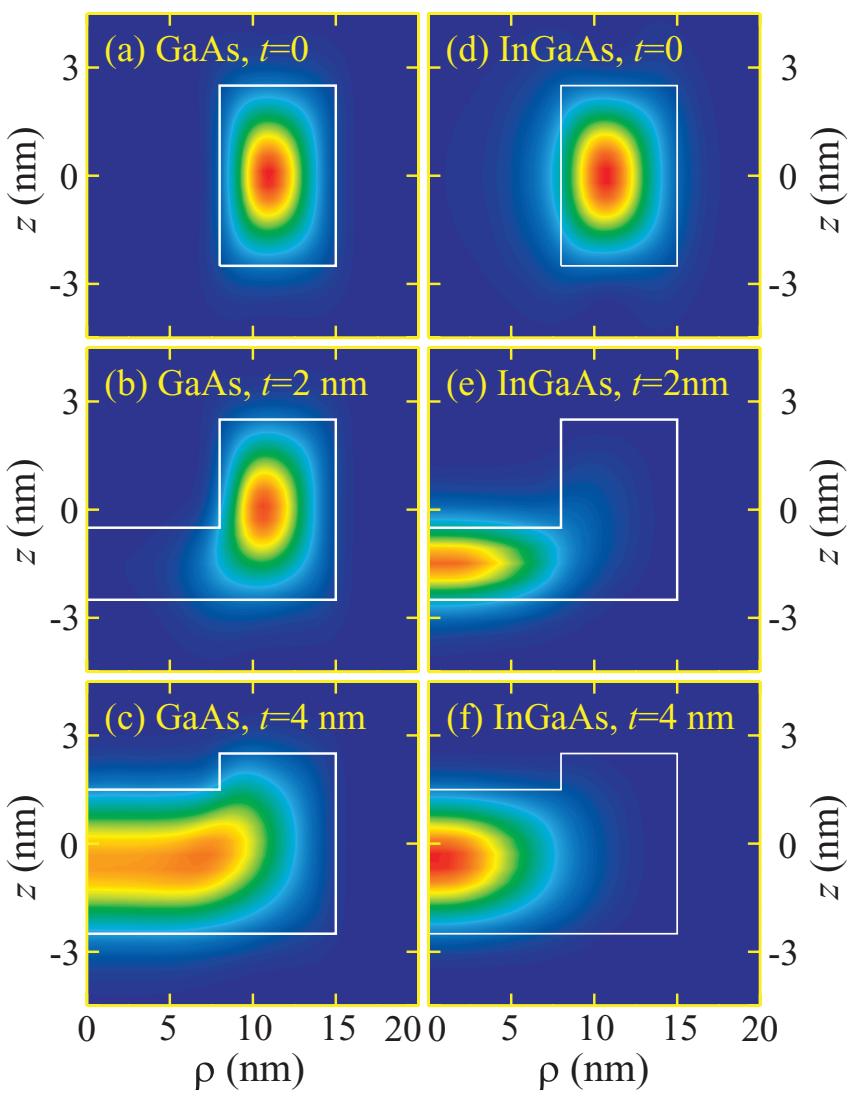

FIG. 6. (Color online) Left panel: The probability density of the single hole $D_{h}$ in the GaAs/(Al,Ga)As nanocup for (a) $t=0$, (b) $t=2 \mathrm{~nm}$, and (c) $t=4 \mathrm{~nm}$. Right panel: $D_{h}$ in the (In,Ga)As/GaAs nanocup for a few values of the disk thickness (d) $t=0$, (e) $t=2 \mathrm{~nm}$, and (f) $t=4 \mathrm{~nm}$.

potential barrier for the light holes is erected inside the disk, the $1 S_{1 / 2}$ state, having the $l=0$ light-hole component, occupies the ring.

In order to illustrate how strain influences the hole localization, we show in Fig. 6 the probability density of holes $\left(D_{h}\right)$ in the ground state for $B=0$. The left panel in this figure [Figs. 6(a)-6(c)] shows $D_{h}$ in the GaAs/(Al,Ga)As ring, and the right panel [Figs. 6(d)-6(f)] displays $D_{h}$ in the (In,Ga)As/GaAs ring. For $t=0$, the hole is mainly localized inside the ring. But, $D_{h}$ in the (In,Ga)As/GaAs nanocup extends more to the ring center, which is a consequence of the confining effective potential for the heavy holes in the ring opening. The presence of the thin disk in the GaAs/(Al,Ga)As nanocup does not considerably affect $D_{h}$, as shown by Fig. 6(b) for $t=2 \mathrm{~nm}$. On the other hand, $V_{h h}$ in the (In,Ga)As/GaAs nanocup confines the hole more inside the disk, which is responsible for the extension of $D_{h}$ towards the cup center, as shown in Fig. 6(e) for $t=2 \mathrm{~nm}$. For $t=4 \mathrm{~nm}$, $D_{h}$ in both nanocups becomes localized inside the disk, as Figs. 6(c) and 6(f) show for the GaAs/(Al,Ga)As and the (In,Ga)As/GaAs nanocups, respectively. However, because of strain, the hole in the (In,Ga)As/GaAs nanocup is practically completely localized around the center of the disk, which leads to the absence of the angular momentum transitions in Fig. 5(c). 

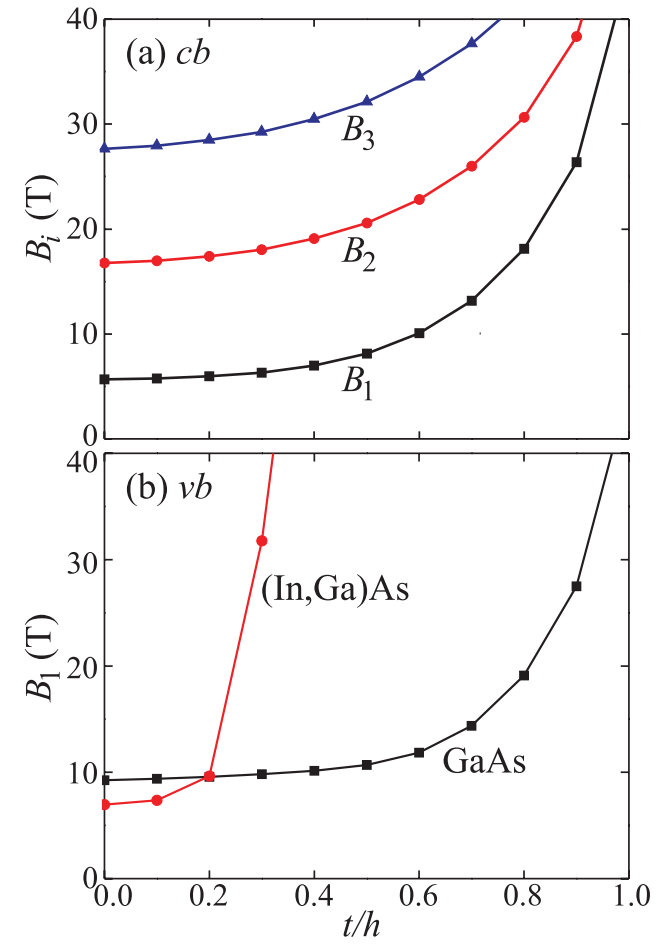

FIG. 7. (Color online) (a) The values of the magnetic fields $B_{1}$, $B_{2}$, and $B_{3}$, where the first three transitions between the orbital momenta of the spin-up electron states take place in the conduction band of the nanocup as a function of the ratio between the disk thickness and the ring height $t / h$. The curves correspond to both the $\mathrm{GaAs} /(\mathrm{Al}, \mathrm{Ga}) \mathrm{As}$ and the (In,Ga)As/GaAs cups. (b) The values of the magnetic field $B_{1}$ for the valence-band states as a function of $t / h$.

\section{Comparisons between the conduction- and valence-band states}

The similarity between the $\mathrm{GaAs} /(\mathrm{Al}, \mathrm{Ga}) \mathrm{As}$ and (In,Ga)As/GaAs systems is due to their equal topology and the similar confining potentials for the electron. Therefore, we show in Fig. 7(a) the dependence of $B_{i}(i=1,2,3)$ with $t / h$ for both systems. As inferred from Fig. $3, B_{i}$ 's are almost constant for $t / h \leqslant 0.4$, i.e., for $t \leqslant 2 \mathrm{~nm}$, but increase rapidly when $t / h$ exceeds 0.4 . One may notice that $B_{1}$ is about three times larger at $t / h=0.8(t=4 \mathrm{~nm})$ than $B_{1}$ for the nanoring $(t=0)$. Besides, all curves in Fig. 7(a) are almost parallel for $t / h<0.6$, which could also have been inferred from Fig. 3 by the nearly parallel thin dashed lines. For $t=h$, when the system becomes a nanodisk, no angular momentum transitions exist for a single electron. Thus, all three curves in Fig. 7(a) tend to infinity when $t / h$ approaches unity.

Because an increase of $t$ leads to both a decrease of the effective potential well for the heavy hole and an increase of the effective potential barrier for the light hole in the disk, the energy difference between the $1 S_{+3 / 2}$ and $1 S_{+1 / 2}$ in the strained (In, Ga)As ring at $B=0$ increases, as Fig. 5 shows. Consequently, the first angular momentum transition between the hole states shifts to much larger values of $B$ than those between the electron states, as Fig. 7(b) displays. Furthermore, $B_{1}$ for the hole in the (In,Ga)As ring increases to a much larger value than $B_{1}$ for the GaAs ring, when $t / h$ increases, as Fig. 7(b) shows.

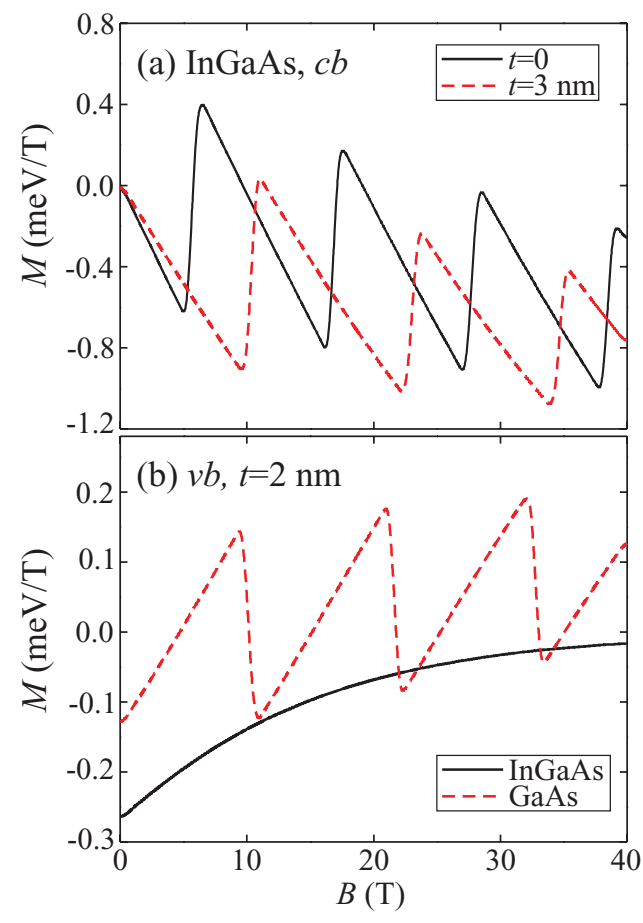

FIG. 8. (Color online) (a) The magnetization of a single electron in the (In,Ga)As/GaAs nanocup for $t=0$ (solid black line) and $t=3 \mathrm{~nm}$ (dashed red line). (b) The magnetization of a single hole in the (In,Ga)As/GaAs (solid black line) and the GaAs/(Al,Ga)As nanocup (dashed red line) for $t=2 \mathrm{~nm}$.

For $t=2 \mathrm{~nm}$, Figs. 3(b) and 5(b) demonstrate that the electron states are affected by the magnetic field as they are confined in the ring, whereas the dependence of the hole ground-state energy level is characteristic of singly connected disks. Therefore, the electron and the hole are spatially separated like in the case of a type-II singly connected nanodot, which is a favorable situation for the appearance of excitonic $\mathrm{AB}$ oscillations. ${ }^{38}$ The calculation of the many-particle exciton states in the nanocup is beyond the scope of this work. However, we infer that in such small structures, the exciton is in the strong confinement regime, ${ }^{39}$ therefore, the $\mathrm{AB}$ oscillations of the exciton are governed by those of the single-particle states.

The peculiar variation of the energy levels with the disk thickness in the strained (In,Ga)As/GaAs nanocup affects the magnetization of the single electron, as Fig. 8(a) displays for $t=0$ and $3 \mathrm{~nm}$. Due to shifts of the orbital momentum transition, the first abrupt step in the $M$ versus $B$ dependence for $t=3 \mathrm{~nm}$ takes place at about $10 \mathrm{~T}$, which is larger by about $4 \mathrm{~T}$ from the value for $t=0$. The dependence of magnetization of the single hole in both systems for $t=$ $2 \mathrm{~nm}$ is shown in Fig. 8(b). Because of the smooth variation of $E_{(1)}^{\text {InGaAs }}$, no oscillations of the hole magnetization are found in the case of the (In,Ga)As/GaAs nanocup, whereas almost periodic oscillations occur for the $\mathrm{GaAs} /(\mathrm{Al}, \mathrm{Ga}) \mathrm{As}$ nanocup. The smooth dependence of $M$ on $B$ in the (In,Ga)As/GaAs nanocup containing a single hole illustrates the previously corroborated fact that the presence of even a thin layer inside the ring opening considerably modifies the electronic structure of the nanoring. 

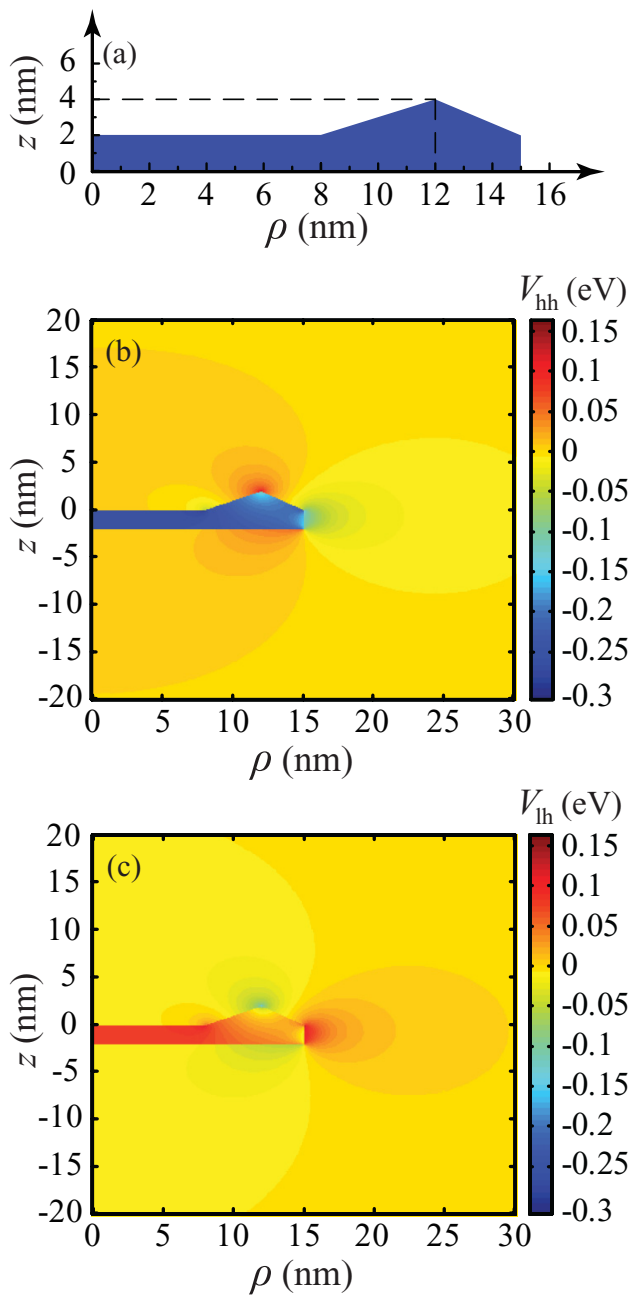

FIG. 9. (Color online) The case of the nanodisk having the bulge of the triangular cross section on the radial periphery (the $\Lambda$-nanocup): (a) the shape and the characteristic dimensions, (b) $V_{h h}$, and (c) $V_{l h}$.

\section{E. Influence of the geometry}

In order to explore how the geometry affects the electron and hole states in the nanocup, we extend our calculations to the strained nanodisks having a bulge of a triangular cross section on its periphery, as shown in Fig. 9(a). Because of the peculiar shape of the rim, we call such a nanodot a $\Lambda$-nanocup, and the previous nanocup of rectangular cross section could be called the $\Pi$-nanocup. The characteristic dimensions of the $\Lambda$-nanocup $R_{1}, R_{2}, t$, and $h$ have similar meaning as for the $\Pi$-nanocup, whereas $R_{p}$ denotes the position of the rim vertex [see Fig. 9(a)]. The shape of this $\Lambda$-nanocup approaches closely the shape of the experimental nanorings, ${ }^{10}$ except that the $\Lambda$-nanocup is axially symmetric, whereas this is not the case for the strained (In,Ga)As/GaAs nanorings explored in Refs. 10 and 13. Furthermore, these nanorings exhibit substantial compositional mixing between the dot and the matrix. Hence, the boundary of the experimental nanorings could not be exactly specified. However, we disregard this effect, and assumed fixed composition of $x=0.5$ in the $\operatorname{In}_{x} \mathrm{Ga}_{1-x}$ As alloy inside the nanocup, and that the matrix is composed of GaAs. ${ }^{13}$ From the available cross section in Refs. 10 and 13 we ex-

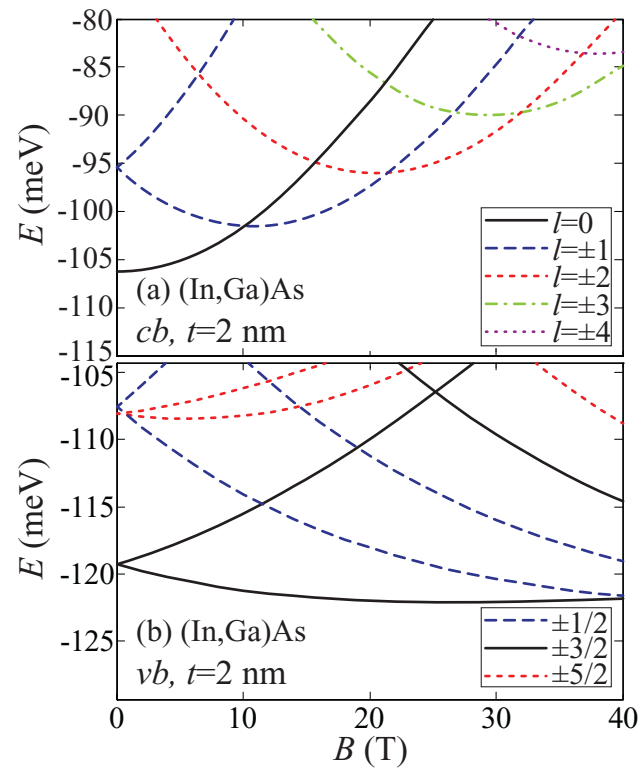

FIG. 10. (Color online) The variations of the electron (a) and the hole (b) energy levels with magnetic field in the $\Lambda$-nanocup.

tracted the following values of the nanocup dimensions: $R_{1}=$ $7 \mathrm{~nm}, R_{2}=15 \mathrm{~nm}, R_{p}=12 \mathrm{~nm}, t=2 \mathrm{~nm}$, and $h=4 \mathrm{~nm}$. The shape of the experimental nanorings is anisotropic in the $x y$ plane, which, in addition to the disk presence, is able to shift the angular momentum transitions in the electron and hole ground-state energy levels with magnetic field. ${ }^{10,13}$ In order to treat the in-plane anisotropy in the nanocup, a 3D model should be employed, which is beyond the scope of this paper.

The effective potential in the heavy-hole band of the $\Lambda$-nanocup is displayed in Fig. 9(b). Similar to the П-nanocup, the effective potential well for the heavy hole is deeper inside the disk than in the rim. Furthermore, inside the rim, the depth of the potential well decreases from the base to the rim vertex. The effective potential in the light-hole band is such that similar to the nanocup, the barrier is erected inside the disk, which is displayed in Fig. 9(c). This barrier is higher in the $\Lambda$-nanocup than in the $\Pi$-nanocup, therefore, it would lead to a smaller contribution of the light-hole states to the hole wave function, and in turn to reduced mixing between the heavy- and light-hole bands. We note that the effective potential in the conduction band, which is not shown in Fig. 9, is qualitatively similar to the one in the П-nanocup (see Fig. 2).

The electron and hole energy levels in the $\Lambda$-nanocup are shown in Figs. 10(a) and 10(b), respectively. For the electron ground state in Fig. 10(a), we determine $B_{1}=10 \mathrm{~T}$, whereas $B_{1}=5.6 \mathrm{~T}$ was previously extracted from the electron ground-state energy level variation with $B$ in Fig. 3(a). The difference between the two values could be explained to arise from smaller volume of the rim in the $\Lambda$-nanocup. Nevertheless, $B_{1}$ for the electron ground state in the $\Lambda$-nanocup is smaller than $14 \mathrm{~T}$, which was the value of $B_{1}$ measured in Ref. 10. The larger value of $B_{1}$ determined in the experiment is a consequence of the in-plane anisotropy of the nanorings explored in Ref. 10, which is not taken into account in our model. The hole ground-state energy level variations in the $\Lambda$ and $\Pi$-nanocups exhibit similar behavior when $B$ varies, i.e., 
they are both continuous in the range [0,40 T] [see Fig. 5(b)]. The small difference between the two could be explained by the deeper effective potential well for the heavy hole in the rim and increased barrier for the light hole inside the disk, as Figs. 9(a) and 9(b) demonstrate. The potential barrier for the light holes leads to a decrease of the light-hole component in the hole ground-state envelope functions. Yet, the changes of the hole energy spectra due to the modified geometry are not large, which implies that geometry of the axially symmetric nanocup is not of a detrimental influence on the appearance and period of the Aharonov-Bohm oscillations in the electron and hole ground-state energy levels.

Finally, we note that our calculations indicate that in strained nanocups, there exists a range of $t$ where the hole is confined similarly as in a disk and the electron as in a ring. Such a localization is advantageous for the appearance of the excitonic $\mathrm{AB}$ oscillations. However, in order to realize such a situation, one should control the thickness of the inner layer during the growth process of strained self-assembled nanorings. The compositional intermixing and the considerable anisotropy of the shape in the $x y$ plane is often present in the currently fabricated nanorings. ${ }^{10,13}$ Previous measurements indicated that the orbital momentum transitions between the electron states are preserved even in the presence of anisotropy, ${ }^{10}$ even though they are shifted by a large amount from the $t=0$ case. More recently, oscillations in the exciton ground-state energy of nearly axially symmetric (In,Ga)As/GaAs nanorings were measured. ${ }^{25}$ Nonetheless, even these nanorings contained a layer inside the nanoring opening.

\section{CONCLUSION}

The hole states in the unstrained GaAs/(Al,Ga)As and the strained (In,Ga)As/GaAs nanocups exhibit oscillations due to angular momentum transitions in a perpendicular magnetic field. These nanocups are composite structures consisting of a nanoring with a disk inside the ring opening. Our calculations show that the angular momentum transitions shift when the disk thickness increases. Because of strain, the hole is preferentially localized inside the disk of the (In,Ga)As/GaAs nanocup, which leads to a large shift of the angular momentum transitions when the disk thickness exceeds already one fifth of ring height. On the other hand, the angular momentum shifts in the valence band of the unstrained $\mathrm{GaAs} /(\mathrm{Al}, \mathrm{Ga})$ As nanocups are small, even for the disk as thick as half the ring height. Similar behavior of the conduction-band states is found in both analyzed systems. Therefore, by increasing the disk thickness in the strained nanocup, the electron ground energy may stay oscillatory (as in the nanoring), whereas the hole ground-state energy level becomes a monotonic function of the magnetic field (as in a singly connected nanodot). Such variations are associated with the dominant localization of the hole inside the disk and the electron in the ring. Therefore, strain could lead to a spatial separation of the electron and the hole, thereby increasing the polarization of the exciton, which is beneficial for the appearance of the excitonic Aharonov-Bohm effect. Furthermore, the detailed geometry of the nanocup is found to have a small effect on the magnetic field dependence of the hole energy levels.

\section{ACKNOWLEDGMENTS}

The authors thank B. Partoens for useful discussions. This work was supported by the EU NoE: SANDiE, the Ministry of Education and Science of Serbia, and the Belgian Science Policy (IAP).

\footnotetext{
*nemanja.cukaric@etf.bg.ac.rs

${ }^{\dagger}$ vladimir.arsoski@etf.bg.ac.rs

${ }^{\ddagger}$ milan.tadic@etf.bg.ac.rs

§francois.peeters@ua.ac.be
}

${ }^{1}$ J. M. García, G. Medeiros-Ribeiro, K. Schmidt, T. Ngo, J. L. Feng, A. Lorke, J. Kotthaus, and P. M. Petroff, Appl. Phys. Lett. 71, 2014 (1997).

${ }^{2}$ A. Lorke, R. J. Luyken, A. O. Govorov, J. P. Kotthaus, J. M. García, and P. M. Petroff, Phys. Rev. Lett. 84, 2223 (2000).

${ }^{3}$ P. Moon, W. J. Choi, K. Park, E. Yoon, and J. Lee, J. Appl. Phys. 109, 103701 (2011).

${ }^{4}$ P. Offermans, P. M. Koenraad, J. H. Wolter, D. Granados, J. M. García, V. M. Fomin, V. N. Gladilin, and J. T. Devreese, Appl. Phys. Lett. 87, 131902 (2005).

${ }^{5}$ T. Mano and N. Koguchi, J. Cryst. Growth 278, 108 (2005).

${ }^{6}$ T. Kuroda, T. Mano, T. Ochiai, S. Sanguinetti, K. Sakoda, G. Kido, and N. Koguchi, Phys. Rev. B 72, 205301 (2005).

${ }^{7}$ C. Somaschini, S. Bietti, S. Sanguinetti, N. Koguchi, and A. Fedorov, Nanotechnology 21, 125601 (2010).

${ }^{8}$ S. Viefers, P. Koskinen, P. Singha Deo, and M. Manninen, Phys. E (Amsterdam) 21, 1 (2004).
${ }^{9}$ B. C. Lee, O. Voskoboynikov, and C. P. Lee, Phys. E (Amsterdam) 24, 87 (2004).

${ }^{10}$ N. A. J. M. Kleemans, I. M. A. Bominaar-Silkens, V. M. Fomin, V. N. Gladilin, D. Granados, A. G. Taboada, J. M. García, P. Offermans, U. Zeitler, P. C. M. Christianen, J. C. Maan, J. T. Devreese, and P. M. Koenraad, Phys. Rev. Lett. 99, 146808 (2007).

${ }^{11}$ M. Tadić, F. M. Peeters, and K. L. Janssens, Phys. Rev. B 65, 165333 (2002).

${ }^{12}$ M. Tadić and F. M. Peeters, Phys. Rev. B 70, 195302 (2004).

${ }^{13}$ V. M. Fomin, V. N. Gladilin, S. N. Klimin, J. T. Devreese, N. A. J. M. Kleemans, and P. M. Koenraad, Phys. Rev. B 76, 235320 (2007).

${ }^{14}$ L. M. Thu, W. T. Chiu, and O. Voskoboynikov, Phys. Rev. B 83, 125301 (2011).

${ }^{15} \mathrm{~S}$. Li and J. Xia, J. Appl. Phys. 91, 3227 (2002).

${ }^{16}$ B. Jia, Z. Yu, and Y. Liu, Modell. Simul. Mater. Sci. Eng. 17, 035004 (2009).

${ }^{17}$ B. Lassen, M. Willatzen, and D. Barettin, Superlattices Microstruct. 47, 103 (2010)

${ }^{18}$ B. Jia, Z. Yu, Y. Liu, W. Yao, H. Feng, and H. Ye, Superlattices Microstruct. 47, 714 (2010).

${ }^{19}$ J. Planelles and W. Jaskólski, J. Phys.: Condens. Matter 15, L67 (2003). 
${ }^{20}$ J. I. Climente, J. Planelles, and W. Jaskólski, Phys. Rev. B 68, 075307 (2003).

${ }^{21}$ A. V. Chaplik, Pis'ma Zh. Eksp. Teor. Fiz. 62, 885 (1995) [JETP Lett. 62, 900 (1995)].

${ }^{22}$ M. Grochol, F. Grosse, and R. Zimmermann, Phys. Rev. B 74, 115416 (2006).

${ }^{23}$ R. Okuyama, M. Eto, and H. Hyuga, Phys. Rev. B 83, 195311 (2011).

${ }^{24}$ T. C. Lin, C. H. Lin, H. S. Ling, Y. J. Fu, W. H. Chang, S. D. Lin, and C. P. Lee, Phys. Rev. B 80, 081304 (2009).

${ }^{25}$ M. D. Teodoro, V. L. Campo, V. Lopez-Richard, E. Marega, G. E. Marques, Y. G. Gobato, F. Iikawa, M. J. S. P. Brasil, Z. Y. AbuWaar, V. G. Dorogan, Y. I. Mazur, M. Benamara, and G. J. Salamo, Phys. Rev. Lett. 104, 086401 (2010).

${ }^{26}$ F. Ding, N. Akopian, B. Li, U. Perinetti, A. Govorov, F. M. Peeters, C. C. Bof Bufon, C. Deneke, Y. H. Chen, A. Rastelli, O. G. Schmidt, and V. Zwiller, Phys. Rev. B 82, 075309 (2010).

${ }^{27}$ M. Tadić, N. Čukarić, V. Arsoski, and F. M. Peeters, Phys. Rev. B 84, 125307 (2011).

${ }^{28}$ J. H. Davies, J. Appl. Phys. 84, 1358 (1998).
${ }^{29}$ M. Tadić, F. M. Peeters, K. L. Janssens, M. Korkusiński, and P. Hawrylak, J. Appl. Phys. 92, 5819 (2002).

${ }^{30}$ N. Čukarić, M. Tadić, and F. M. Peeters, Superlattices Microstruct. 48, 491 (2010).

${ }^{31}$ J. I. Climente, J. Planelles, and J. L. Movilla, Phys. Rev. B 70, 081301 (2004).

${ }^{32}$ F. B. Pedersen and Y.-C. Chang, Phys. Rev. B 53, 1507 (1996).

${ }^{33}$ CalvinYi-Ping Chao and S. L. Chuang, Phys. Rev. B 46, 4110 (1992).

${ }^{34}$ J. Planelles, J. I. Climente, F. Rajadell, M. F. Doty, A. S. Bracker, and D. Gammon, Phys. Rev. B 82, 155307 (2010).

${ }^{35}$ I. Vurgaftman, J. R. Meyer, and L. R. Ram-Mohan, J. Appl. Phys. 89, 5815 (2001).

${ }^{36}$ P. Lawaetz, Phys. Rev. B 4, 3460 (1971).

${ }^{37}$ M. Bayer, A. Kuther, A. Forchel, A. Gorbunov, V. B. Timofeev, F. Schäfer, J. P. Reithmaier, T. L. Reinecke, and S. N. Walck, Phys. Rev. Lett. 82, 1748 (1999).

${ }^{38}$ E. Ribeiro, A. O. Govorov, W. Carvalho Jr., and G. MedeirosRibeiro, Phys. Rev. Lett. 92, 126402 (2004).

${ }^{39}$ A. O. Govorov, S. E. Ulloa, K. Karrai, and R. J. Warburton, Phys. Rev. B 66, 081309 (2002). 